\title{
D-decomposition technique for stabilization of Furuta pendulum: fractional approach
}

\author{
P.D. MANDIĆ ${ }^{1 *}$, M.P. LAZAREVIĆ ${ }^{1}$, and T.B. ŠEKARA ${ }^{2}$ \\ ${ }^{1}$ Faculty of Mechanical Engineering, Department of Mechanics, University of Belgrade, 16 Kraljice Marije St., 11120 Belgrade, Serbia \\ ${ }^{2}$ School of Electrical Engineering, Signals and Systems Department, University of Belgrade, \\ 73 Bulevar Kralja Aleksandra St., 11120 Belgrade, Serbia
}

\begin{abstract}
In this paper, the stability problem of Furuta pendulum controlled by the fractional order PD controller is presented. A mathematical model of rotational inverted pendulum is derived and the fractional order PD controller is introduced in order to stabilize the same. The problem of asymptotic stability of a closed loop system is solved using the D-decomposition approach. On the basis of this method, analytical forms expressing the boundaries of stability regions in the parameters space have been determined. The D-decomposition method is investigated for linear fractional order systems and for the case of linear parameter dependence. In addition, some results for the case of nonlinear parameter dependence are presented. An example is given and tests are made in order to confirm that stability domains have been well calculated. When the stability regions have been determined, tuning of the fractional order PD controller can be carried out.
\end{abstract}

Key words: fractional order PID, D-decomposition, asymptotic stability, inverted pendulum.

\section{Introduction}

The D-decomposition technique is an extremely powerful method used in control theory for investigation of linear systems asymptotic stability. It is particularly valuable when a system has to be synthetized by determining the values of control parameters based on some performance criteria. This technique has been first introduced by Neimark [1,2] and recognized in Russian literature as the method of principal interest, while in Western Europe and the USA it has attracted little interest. The method is based on the following idea. Consider for example, a characteristic equation in general form:

$$
f(s)=a_{n} s^{b_{n}}+\ldots+a_{k} s^{b_{k}}+\ldots+a_{0} s^{b_{0}}=0,
$$

where the coefficients $a_{k}$ and $b_{k}(k=0,1, \ldots, n)$ are nonlinear functions of system parameters $\alpha$ and $\beta$, and $b_{0}<b_{1}<$ $\cdots<b_{n}$. If $s$ is expressed as $s=j \omega$ where $\omega$ represents frequency, characteristic equation (1) may be rewritten as two equations in two unknowns $\alpha$ and $\beta$. In other words, applying conditions that the summations of reals and imaginaries must go to zero independently, (1) can be specified in terms of its real $R$ and imaginary part $I$ as follows:

$$
R=R(\omega, \alpha, \beta)=0, \quad I=I(\omega, \alpha, \beta)=0 .
$$

If coefficients $a_{k}$ and $b_{k}$ are linear functions of parameters $\alpha$ and $\beta$, Eq. (2) may be solved for $\alpha$ and $\beta$ as:

$$
\alpha=\alpha(\omega), \quad \beta=\beta(\omega) .
$$

In general, when parameters $\alpha$ and $\beta$ form a nonlinear relationship, the solution (3) is not possible in a closed form, and can be solved using numerical methods. Equations (3) are used to map imaginary axis from the $s$-plane onto the $(\alpha, \beta)$ parameters plane. In this mapping boundaries are obtained, which divide the $(\alpha, \beta)$ parameter plane into a number of domains with a fixed root distribution. Then, the number of roots in each of the bounded regions can be easily determined, including those domains corresponding to the conditions of asymptotic stability.

Control of underactuated mechanical systems is currently an active research field and it has attracted a lot of interest in the control community in the last years. Underactuated systems are the control systems with fewer control inputs than degrees of freedom. This lack of inputs is the main difficulty in controlling them. Rotational inverted pendulum is one of the most well-known underactuated mechanical systems [3]. It is an excellent benchmark for testing different control algorithms because it represents a highly nonlinear system and unstable at the desired upright position. One of the goals of this paper is stabilization of the inverted pendulum using fractional order controller.

Fractional calculus (FC) is a mathematical topic with more than 300 years old history, but its application to physics and engineering has been reported only in the recent years. Fractional integrate-differential operators are a generalization of integration and derivation to non-integer order (fractional) operators [4-6]. As we know, due to their functional simplicity and performance robustness, the PID controllers are still used for many industrial applications. On the other hand, fractional calculus has the potential to accomplish what an integerorder calculus cannot. In most cases, our objective of using fractional calculus is to apply the fractional order controller to enhance system performance compared to the traditional controllers. Fractional $P I^{\beta} D^{\alpha}$ controller [7], CRONE controllers, and fractional lead-lag compensator, are some of the well-known fractional order controllers.

*e-mail: pmandic@mas.bg.ac.rs 
In this paper, the fractional order PD controller is used for stabilization of rotational inverted pendulum. The Ddecomposition technique is used for determining stability regions in the controller parameters space. This method is applied to linear fractional systems, and for the case of linear parameters dependence. For calculating regions of asymptotic stability an efficient computational method is developed. Once the stability domains are determined, tuning of the fractional order controller can be continued with.

The rest of the paper is organized as follows. A mathematical model of rotational inverted pendulum has been shown in Sec. 2, and in Sec. 3 a fractional order PD controller has been introduced in order to stabilize it. Section 4 presents the D-decomposition method for calculation of stability regions in the controller parameters space. Examples have been provided in Sec. 5 and tests with different controller parameters have been simulated and analyzed. Section 6 concludes the paper.

\section{Mathematical model}

Figure 1 depicts a schematic view of the rotational inverted pendulum, which is also known in literature as Furuta pendulum. It consists of horizontal arm and vertical pendulum, angular positions of which are denoted as $\theta$ and $\varphi$, respectively. Hence, it is a mechanical system with two degrees of freedom. Torque is applied directly to the arm, while no torque drives the pendulum. This lack of control input makes Furuta pendulum an underactuated mechanical system difficult to control. The system has two outputs $(\theta$ and $\varphi)$, one control signal (external torque $M$ ), and four state variables represented by state vector $x=[\theta, \dot{\theta}, \varphi, \dot{\varphi}]^{T}$.

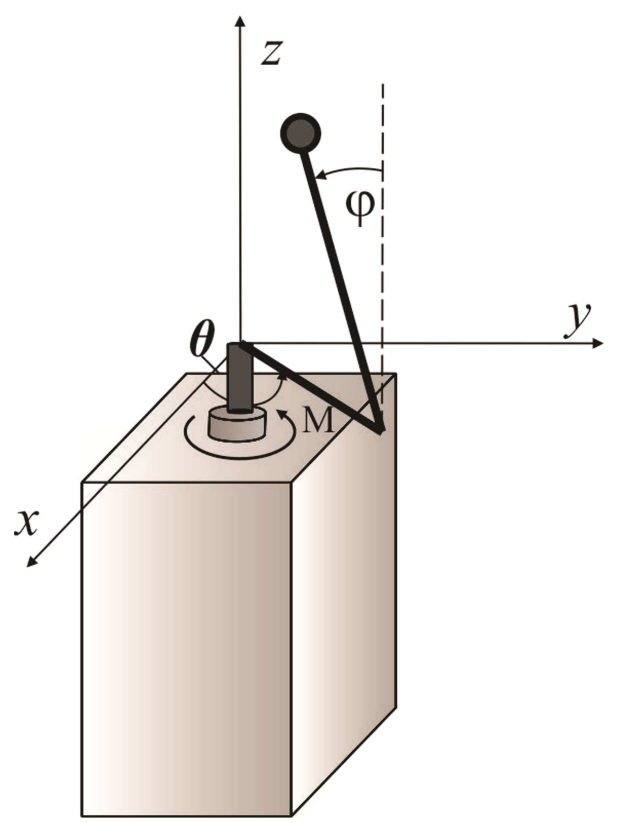

Fig. 1. A schematic view of the Furuta pendulum

The system parameters are as following: $m_{1}-$ mass of the arm, $m_{2}-$ mass of the pendulum, $R_{1}$ - distance of the arm pivot point to the pendulum pivot point, $R_{2}$ - distance of the pendulum pivot point to its end (extreme), $2 r_{1}, 2 r_{2}-$ total length of the arm, and pendulum respectively, $J_{\varsigma 1}-$ moment of inertia of the arm with respect to its centre of mass, $J_{\xi 2}, J_{\eta 2}, J_{\zeta 2}$ - axial moments of inertia of the pendulum with respect to its centre of mass.

Rodriguez method [8,9] is suggested for modelling the system dynamics. Configuration of mechanical model can be defined by the generalized coordinates $q_{1}$ and $q_{2}$ represented by $\theta$ and $\varphi$, respectively. Herein, Langrange's equations of the second kind expressed in a covariant form are used as the equations of motion of the inverted pendulum [3]:

$$
\sum_{\alpha=1}^{n} a_{\gamma \alpha} \ddot{q}_{\alpha}+\sum_{\alpha=1}^{n} \sum_{\beta=1}^{n} \Gamma_{\alpha \beta, \gamma} \dot{q}_{\alpha} \dot{q}_{\beta}=Q_{\gamma}, \quad \gamma=1,2 .
$$

The coefficients $a_{\alpha \beta}$ represent covariant coordinates of the basic metric tensor $\left[a_{\gamma \alpha}\right] \in R^{2 \times 2}$ and $\Gamma_{\alpha \beta, \gamma} \alpha, \beta, \gamma=1,2$ presents Christoffel symbols of the first kind. The right side of (4) denotes generalized spring forces, gravitational forces, semi-dry friction and generalized control forces [10]. The generalized forces $Q_{\gamma}$ can be expressed as:

$$
Q_{\gamma}=Q_{\gamma}^{g}+Q_{\gamma}^{a}, \quad \gamma=1,2,
$$

wherein $Q_{\gamma}^{g}, Q_{\gamma}^{a}$ represent the generalized gravitational and control forces, respectively. Now, the equations of motion of Furuta pendulum can be rewritten in full form:

$$
\begin{gathered}
a_{11} \ddot{\theta}+a_{12} \ddot{\varphi}+2 \Gamma_{12,1} \dot{\theta} \dot{\varphi}+\Gamma_{22,1} \dot{\varphi}^{2}=Q_{1}^{a}, \\
a_{12} \ddot{\theta}+a_{22} \ddot{\varphi}-\Gamma_{12,1} \dot{\theta}^{2}=Q_{2}^{g},
\end{gathered}
$$

wherein

$$
\begin{gathered}
a_{11}=J_{\varsigma 1}+J_{\eta 2} \sin ^{2}(\varphi)+J_{\varsigma 2} \cos ^{2}(\varphi)+m_{2} R_{1}^{2} \\
+m_{1}\left(R_{1}-r_{1}\right)^{2}+m_{2}\left(R_{2}-r_{2}\right)^{2} \sin ^{2}(\varphi), \\
a_{12}=-m_{2} R_{1}\left(R_{2}-r_{2}\right) \cos (\varphi)=-K_{3} \cos (\varphi), \\
a_{22}=J_{\xi 2}+m_{2}\left(R_{2}-r_{2}\right)^{2}=K_{4}, \\
\Gamma_{12,1}=0.5\left(m_{2}\left(R_{2}-r_{2}\right)^{2}+J_{\eta 2}-J_{\varsigma 2}\right) \sin (2 \varphi) \\
=K_{2} \sin (2 \varphi), \\
\Gamma_{22,1}=m_{2} R_{1}\left(R_{2}-r_{2}\right) \sin (\varphi)=K_{3} \sin (\varphi), \\
Q_{2}^{g}=m_{2} g\left(R_{2}-r_{2}\right) \sin (\varphi)=K_{1} \sin (\varphi), \\
Q_{1}^{a}=M .
\end{gathered}
$$

The external torque which drives the arm is denoted as $M$, as shown above. Physical parameters $K_{1}, K_{2}, K_{3}, K_{4}$ are introduced in (8) to simplify writing of the equations of motion.

The Furuta pendulum has an infinite number of equilibrium points (in downward and upright positions) [11]. Stable downward equilibrium points correspond to $\pi$ pendulum angle and zero angular velocities $\left(x_{e 1}=[\theta, 0, \pi, 0]^{T}\right)$. Unstable upright equilibrium points correspond to zero pendulum angle and angular velocities $\left(x_{e 2}=[\theta, 0,0,0]^{T}\right)$. As we can see, equilibrium points exist for $\forall \theta \in \mathbb{R}$. The goal of this 


\section{D-decomposition technique for stabilization of Furuta pendulum: fractional approach}

paper is stabilization of pendulum around its unstable equilibrium point, with additional condition $\theta=0$, which makes stabilization problem more difficult to solve [12].

\section{Controller design}

Control strategy for stabilization of the inverted pendulum usually consists of two different problems. The first one is swinging phase, where one needs to steer the pendulum from the down to the upright position. Energy control techniques are usually used for solving this problem. When the pendulum is close enough to the upright position, balancing controller takes over the control and stabilizes the pendulum. The goal of this paper is stabilization of the pendulum and finding its stability domains using the D-decomposition method. Hence, control design for the swing up controller will not be consedered here.

Firstly, we show the simplification of the dynamic equations of the inverted pendulum using partial feedback linearization [11]. If we calculate $\ddot{\theta}$ from (7) and plug it into (6), the following is obtained:

$$
\begin{gathered}
\frac{a_{11}}{a_{12}}\left(Q_{2}^{g}+\Gamma_{12,1} \dot{\theta}^{2}\right)+\left(a_{12}-\frac{a_{11} a_{22}}{a_{12}}\right) \ddot{\varphi} \\
+2 \Gamma_{12,1} \dot{\theta} \dot{\varphi}+\Gamma_{22,1} \dot{\varphi}^{2}=M .
\end{gathered}
$$

Eliminating $\ddot{\theta}$ from (9), the control input $M$ can now be chosen as:

$$
\begin{gathered}
M=\frac{a_{11}}{a_{12}}\left(Q_{2}^{g}+\Gamma_{12,1} \dot{\theta}^{2}\right)+\left(a_{12}-\frac{a_{11} a_{22}}{a_{12}}\right) M_{R} \\
+2 \Gamma_{12,1} \dot{\theta} \dot{\varphi}+\Gamma_{22,1} \dot{\varphi}^{2},
\end{gathered}
$$

wherein $M_{R}$ stands for new control signal. Now, the simplified dynamic equations of Furuta pendulum are as follows:

$$
\begin{gathered}
\ddot{\theta} \cos (\varphi)=-\frac{K_{1}}{K_{3}} \sin (\varphi)-\frac{K_{2}}{K_{3}} \sin (2 \varphi) \dot{\theta}^{2}+\frac{K_{4}}{K_{3}} M_{R}, \\
\ddot{\varphi}=M_{R},
\end{gathered}
$$

wherein the parameters $K_{1}, K_{2}, K_{3}, K_{4}$ are defined in (8). Figure 2 illustrates in block diagram form the idea of partial feedback linearization.

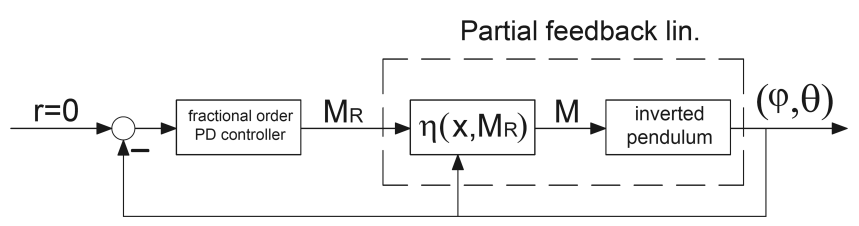

Fig. 2. Partial feedback linearization procedure

A nonlinear feedback of the form $M=\eta\left(x, M_{R}\right)$ is used to modify the dynamics of nonlinear process so that the response from the input $M_{R}$ to the output $\varphi$ is linear. A linear fractional PD controller can then be used to regulate the system dynamics.

Now, to explore the behavior of the system described with (11), (12) near the equilibrium point $(\theta, \dot{\theta}, \varphi, \dot{\varphi})=(0,0,0,0)$ we will linearize the system. Taylor series expansion of nonlinear terms in Eq. (11) around the equilibrium gives:

$$
\begin{array}{r}
f_{1}=\left.\cos (\varphi) \approx \cos (\varphi)\right|_{0}+\left.(\varphi-0) \frac{d f_{1}}{d \varphi}\right|_{0}=1, \\
f_{2}=\left.\sin (2 \varphi) \dot{\theta}^{2} \approx \sin (2 \varphi) \dot{\theta}^{2}\right|_{0,0} \\
+\left.(\varphi-0) \frac{\partial f_{2}}{\partial \varphi}\right|_{0,0}+\left.(\dot{\theta}-0) \frac{\partial f_{2}}{\partial \dot{\theta}}\right|_{0,0}=0, \\
f_{3}=\left.\sin (\varphi) \approx \sin (\varphi)\right|_{0}+\left.(\varphi-0) \frac{d f_{3}}{d \varphi}\right|_{0}=\varphi .
\end{array}
$$

So, the linearization around desired equilibrium point leads to:

$$
\begin{gathered}
\ddot{\theta}=-\frac{K_{1}}{K_{3}} \varphi+\frac{K_{4}}{K_{3}} M_{R}, \\
\ddot{\varphi}=M_{R} .
\end{gathered}
$$

We can see that both Eqs. (16) and (17) are coupled by term $M_{R}$, which complicates the control design for our system. The system (16), (17) approximates the original system (11), (12) when we are near the equilibrium point about which the system was linearized. Using Lyapunov's indirect method theorem [11], if the linearization is asymptotically stable, then the equilibrium point is locally asymptotically stable for the full nonlinear system. Other words, the controller derived from a linearized system will work for a nonlinear system if the region of attraction is not too large.

Asymptotic stability for $(\varphi, \dot{\varphi})$ can be achieved with a simple PD controller of the form $M_{R}=-K_{P \varphi} \varphi-K_{D \varphi} \dot{\varphi}$. It stabilizes the pendulum for $K_{P \varphi}, K_{D \varphi}>0$, but it does not stabilize the arm. The reason for this is that actuated subsystems (16) is coupled with the unactuated subsystem (17) through the control input $M_{R}$. This is why underactuated systems such as the inverted pendulum are difficult to implement a full feedback linearization procedure. Therefore, the new goal of the controller design is to improve $M_{R}$ so that the asymptotic stability for $(\varphi, \dot{\varphi}, \theta, \dot{\theta})$ can be achieved. To accomplish this, an extended fractional order PD controller is suggested. It is a generalization of conventional PD controller $[13,14]$, control feedback law of which is extended as follows: $M_{R}=-\left(K_{P \theta} \theta+K_{D \theta} \theta^{\alpha}\right)-\left(K_{P \varphi} \varphi+K_{D \varphi} \varphi^{\beta}\right)+\frac{K_{1}}{K_{4}} \varphi$,

wherein $\alpha, \beta$ denote real differentiation parameters, and $K_{P \theta}$, $K_{D \theta}, K_{P \varphi}, K_{D \varphi}$ proportional and differential gains of the controller. After substituting (18) into (16) and (17), the dynamic equations of the inverted pendulum become:

$$
\begin{aligned}
& \ddot{\theta}+\frac{K_{4}}{K_{3}} K_{D \theta} \theta^{\alpha}+\frac{K_{4}}{K_{3}} K_{P \theta} \theta=-\frac{K_{4}}{K_{3}} K_{D \varphi} \varphi^{\beta}-\frac{K_{4}}{K_{3}} K_{P \varphi} \varphi, \\
& \ddot{\varphi}+K_{D \varphi} \varphi^{\beta}+\left(K_{P \varphi}-\frac{K_{1}}{K_{4}}\right) \varphi=-K_{D \theta} \theta^{\alpha}-K_{P \theta} \theta .
\end{aligned}
$$

The last term on the right side of (18) is introduced to cancel out the term which contains $\varphi$ in (16). The classical PD controller is obtained for $\alpha=\beta=1$. Four controller gains 
P.D. Mandić, M.P. Lazarević, and T.B. Šekara

$\left(K_{P \theta}, K_{D \theta}, K_{P \varphi}, K_{D \varphi}\right)$ and two additional orders $(\alpha, \beta)$ can be changed in order to achieve either asymptotic or relative stability of the closed loop system. In this paper, the influence of $K_{D \theta}, K_{D \varphi}, \alpha$ and $\beta$ parameters on the asymptotic stability of the system (19), (20) is investigated using the D-decomposition method.

\section{4. D-decomposition method for stabilization of Furuta pendulum}

Using the classical D-decomposition method $[1,2]$ the stability domain in the parameter plane $\left(K_{D \theta}, K_{D \varphi}\right)$ can be calculated. These two parameters form linear relationship, and because of this property analytical form expressing the boundaries of stability region can be calculated. Some results of the D-decomposition procedure for robotic systems have been given in [15]. A characteristic polynomial of the system described with (19), (20) is given by:

$$
\begin{gathered}
f(s)=s^{4}+s^{2}\left(K_{4} K_{D \theta} s^{\alpha} / K_{3}+K_{D \varphi} s^{\beta}\right) \\
-K_{1} K_{D \theta} s^{\alpha} / K_{3} \\
+s^{2}\left(K_{4} K_{P \theta} / K_{3}+K_{P \varphi}-K_{1} / K_{4}\right) \\
-K_{1} K_{P \theta} / K_{3} .
\end{gathered}
$$

The parameter plane $\left(K_{D \theta}, K_{D \varphi}\right)$ is decomposed by the boundaries of D-decomposition into a finite number regions $\mathrm{D}(k)$. Any point in domain $\mathrm{D}(k)$ corresponds to such values of $K_{D \theta}$ and $K_{D \varphi}$ that polynomial (21) has exactly $k$ zeroes with positive real parts [16]. The region $\mathrm{D}(0)$ represents the region of asymptotic stability of the system. Stability boundaries are curves on which each point corresponds to characteristic equation having solutions on the imaginary axes. It can be real zero boundary, complex zero boundary, or singular line [17].

A real zero boundary is determined by the equation $f(0)=0$. It follows that polynomial (21) has no zero $s=0$ if $K_{P \theta} \neq 0$, which will be the case in this paper. Otherwise, the system is structurally unstable. Complex zero boundary corresponds to the pure imaginary zeroes of characteristic polynomial. We can obtain this boundary by solving the equation:

$$
\begin{gathered}
w^{4}-w^{2}\left(K_{4} K_{D \theta}(j w)^{\alpha} / K_{3}+K_{D \varphi}(j w)^{\beta}\right) \\
-K_{1} K_{D \theta}(j w)^{\alpha} / K_{3} \\
-w^{2}\left(K_{4} K_{P \theta} / K_{3}+K_{P \varphi}-K_{1} / K_{4}\right) \\
-K_{1} K_{P \theta} / K_{3}=0
\end{gathered}
$$

which we get by substituting $s=j w$ in polynomial (21) and equating it to 0 . Complex equation (22) can be rewritten as [18]:

$$
f(j w)=u(w, \alpha, \beta)+j v(w, \alpha, \beta)=0,
$$

where $u(w, \alpha, \beta)$ and $v(w, \alpha, \beta)$ denote the real and imaginary part of (22). Terms $(j w)^{\alpha}$ and $(j w)^{\beta}$ which are required for (22) can be expressed as [19]:

$$
(j w)^{\alpha}=w^{\alpha}(\cos (\alpha \pi / 2)+j \sin (\alpha \pi / 2)), \quad w \geq 0 .
$$

Then, equating the real and imaginary part of (23) to zero, one obtains the following 2-D system:

$$
\left[\begin{array}{cc}
U_{1}(w, \alpha, \beta) & U_{2}(w, \alpha, \beta) \\
V_{1}(w, \alpha, \beta) & V_{2}(w, \alpha, \beta)
\end{array}\right]\left(\begin{array}{c}
K_{D \theta} \\
K_{D \varphi}
\end{array}\right)=\left(\begin{array}{l}
Q_{1}(w) \\
Q_{2}(w)
\end{array}\right),
$$

wherein

$$
\begin{gathered}
a=K_{4} / K_{3}, \quad b=K_{1} / K_{3}, \\
U_{1}(w, \alpha, \beta)=\left(a w^{2}+b\right) w^{\alpha} \cos (0.5 \alpha \pi), \\
U_{2}(w, \alpha, \beta)=w^{2+\beta} \cos (0.5 \beta \pi), \\
V_{1}(w, \alpha, \beta)=\left(a w^{2}+b\right) w^{\alpha} \sin (0.5 \alpha \pi), \\
V_{2}(w, \alpha, \beta)=w^{2+\beta} \sin (0.5 \beta \pi), \\
Q_{1}(w)=w^{4}-w^{2}\left(a K_{P \theta}+K_{P \varphi}-b / a\right)-b K_{P \theta}, \\
Q_{2}(w)=0 .
\end{gathered}
$$

Solving it for parameters $\left(K_{D \theta}, K_{D \varphi}\right)$, we obtain [20]:

$$
K_{D \theta}=\frac{\Delta_{\theta}}{\Delta}, \quad K_{D \varphi}=\frac{\Delta_{\varphi}}{\Delta},
$$

wherein

$$
\begin{aligned}
& \Delta=\left|\begin{array}{cc}
U_{1}(w, \alpha, \beta) & U_{2}(w, \alpha, \beta) \\
V_{1}(w, \alpha, \beta) & V_{2}(w, \alpha, \beta)
\end{array}\right|, \\
& \Delta_{\theta}=\left|\begin{array}{cc}
Q_{1}(w) & U_{2}(w, \alpha, \beta) \\
0 & V_{2}(w, \alpha, \beta)
\end{array}\right|, \\
& \Delta_{\varphi}=\left|\begin{array}{cc}
U_{1}(w, \alpha, \beta) & Q_{1}(w) \\
V_{1}(w, \alpha, \beta) & 0
\end{array}\right| .
\end{aligned}
$$

It can be easily shown that:

$$
\Delta=\left(a w^{2}+b\right) w^{\alpha+\beta+2} \sin (0.5(\beta-\alpha) \pi) .
$$

For $\Delta \neq 0,(27)$ describe a curve in the $\left(K_{D \theta}, K_{D \varphi}\right)$ parameter plane representing the complex zero boundary, for constant values $K_{P \theta}, K_{P \varphi}, \alpha$ and $\beta$, as $w$ runs from 0 to $\infty$. In crossing this curve, two roots in $s$-plane move from one half plane to another.

A more detailed analysis must be done when $\Delta=0$. It follows from (30) that this is true for $w=0$ or $\beta-\alpha=$ $k, \forall k=0, \pm 2, \pm 4, \ldots$. For the first case when $w=0$, (26) can be written as:

$$
\begin{gathered}
U_{1}(0, \alpha, \beta)=0, \quad U_{2}(0, \alpha, \beta)=0, \quad Q_{1}(0)=-b K_{P \theta}, \\
V_{1}(0, \alpha, \beta)=0, \quad V_{2}(0, \alpha, \beta)=0, \quad Q_{2}(w)=0 .
\end{gathered}
$$

It follows from (25) and (31) that $0=-b K_{P \theta}$. This is not true for $K_{P \theta} \neq 0$, so the system (25) has no real solutions for $w=0$. In the second case, $\Delta=0$ for $\beta-\alpha=k$, $\forall k=0, \pm 2, \pm 4, \ldots$. By limiting the parameters $\alpha$ and $\beta$ to be in range of $(0,2)$, it follows $\beta-\alpha=0$. Now, for $\beta=\alpha$, (26) reads: 


$$
\begin{gathered}
U_{1}(w, \alpha, \alpha)=\left(a w^{2}+b\right) w^{\alpha} \cos (0.5 \alpha \pi), \\
U_{2}(w, \alpha, \alpha)=w^{2+\alpha} \cos (0.5 \alpha \pi), \\
V_{1}(w, \alpha, \alpha)=\left(a w^{2}+b\right) w^{\alpha} \sin (0.5 \alpha \pi), \\
V_{2}(w, \alpha, \alpha)=w^{2+\alpha} \sin (0.5 \alpha \pi), \\
Q_{1}(w)=w^{4}-w^{2}\left(a K_{P \theta}+K_{P \varphi}-b / a\right)-b K_{P \theta}, \\
Q_{2}(w)=0 .
\end{gathered}
$$

Equation (25) can be expressed as:

$$
\begin{gathered}
w^{\alpha} \cos (0.5 \alpha \pi)\left[\left(a w^{2}+b\right) K_{D \theta}+w^{2} K_{D \varphi}\right] \\
=w^{4}-w^{2}\left(a K_{P \theta}+K_{P \varphi}-b / a\right)-b K_{P \theta}, \\
\left(a w^{2}+b\right) K_{D \theta}+w^{2} K_{D \varphi}=0 .
\end{gathered}
$$

Equations (33), (34) are satisfied when:

$$
d(w)=w^{4}-w^{2}\left(a K_{P \theta}+K_{P \varphi}-b / a\right)-b K_{P \theta}=0 .
$$

Frequency $w_{s}$ for which $d\left(w_{s}\right)=0$ determines a singular line. In this case $\Delta=\Delta_{\theta}=\Delta_{\varphi}=0$, and D-decomposition contains not a point, but a whole line. This singular line can be obtained from either (33) or (34), and it reads:

$$
K_{D \varphi}=-\left(a+\frac{b}{w_{s}^{2}}\right) K_{D \theta} .
$$

Stability boundaries in $\left(K_{D \theta}, K_{D \varphi}\right)$ parameter space are determined by (27) and (36). Now, an example will be given in order to confirm that stability domains are well calculated.

\section{Simulation results}

In this section, numerical simulations of the proposed method have been presented. Physical parameters of the system $K_{1}$, $K_{2}, K_{3}, K_{4}$ are taken from the real laboratory model of Furuta pendulum, and have the following values: $K_{1}=6.51 \cdot 10^{-2}$, $K_{2}=9.18 \cdot 10^{-4}, K_{3}=1.42 \cdot 10^{-3}$, and $K_{4}=1.83 \cdot 10^{-3}$. Using the D-decomposition method, stable and unstable regions in parameter space $\left(K_{D \theta}, K_{D \varphi}\right)$ are obtained. Checking one arbitrary test point within each region, and testing the stability of polynomial (21) using the inverse Laplace transformation, one can find the domains of asymptotic stability of the system described with (19), (20). To simplify, in this simulation only the stability region $\mathrm{D}(0)$ has been presented.

Equations (27) and (36) determine the stability boundaries in parameter space $\left(K_{D \theta}, K_{D \varphi}\right)$. For constant $K_{P \theta}, K_{P \varphi}, \alpha$ and $\beta$, stability region in $\left(K_{D \theta}, K_{D \varphi}\right)$ parameter space is calculated. Practically, instead of $\left(K_{D \theta}, K_{D \varphi}\right)$ we could choose any other two out of these six parameters and examine their influence on asymptotic stability of the system, but covering all the cases could not be done herein due to limitations in paper size. Still, it is interesting to briefly consider what happens when fractional derivatives are taken for parameters instead of $\left(K_{D \theta}, K_{D \varphi}\right)$. In this case, if we choose to determine the influence of $\alpha$ and $\beta$ parameters on system stability, two implicit, nonlinear equations are obtained analytical solution of which is very difficult to find. Solving this task is beyond the scope of paper, and reader is referred to [14] where special attention is given to this problem, with alternative Ddecomposition procedure suggested for resolving it. However, the influence of $\alpha$ and $\beta$ parameters on the system stability can still be investigated herein indirectly using the procedure explained in previous section. More specifically, repeating the D-decomposition method in $\left(K_{D \theta}, K_{D \varphi}\right)$ parameters space, but for different values of $\alpha$ parameter (while $\beta$ remains constant), stability region in $\left(K_{D \theta}, K_{D \varphi}, \alpha\right)$ parameters plane can be obtained. This way, two qualitative improvements are achieved:

- D-decomposition method is extended from twodimensional to three-dimensional parameters space and,

- D-decomposition method for linear parameter dependence is extended for the case of nonlinear parameter dependence, without solving directly nonlinear equations.

Now, the influence of $\left(K_{D \theta}, K_{D \varphi}, \alpha\right)$ parameters on the system stability can be investigated using the Ddecomposition approach. Controller gains $K_{P \theta}$ and $K_{P \varphi}$ are assumed to be known and chosen as: $K_{P \theta}=-0.022$, $K_{P \varphi}=41.5$, while $\beta=1$. For the case $\alpha \in(0,2)$ the stability regions have been plotted and depicted in Fig. 3.

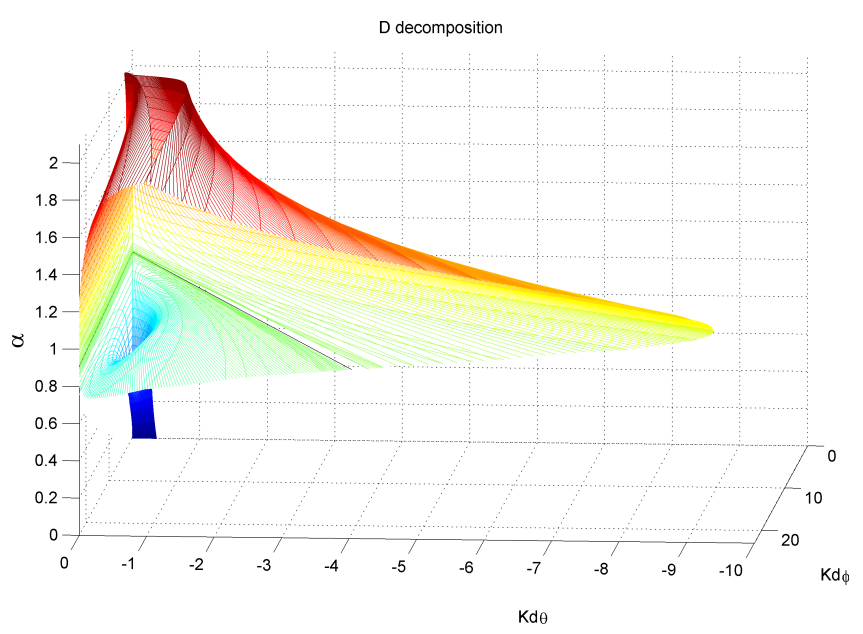

Fig. 3. 3D Stability regions for $\alpha=(0,2), \beta=1$

It is noted that different choices of $\alpha$ lead to different stability regions. The value of $\alpha$ giving the biggest stability region can be chosen. Picking a point deep inside stability region we ensure that system is more robust with respect to parameter variations, which implies bigger stability margins. Global stability region in a 2D plot is shown in Fig. 4.

For $\alpha=\beta=1$ stability region is determined only with singular lines:

$$
K_{D \varphi}=-267.2 K_{D \theta}, \quad K_{D \varphi}=-9.11 K_{D \theta} .
$$

Now, by varying $\beta$ from 0 to 2 and repeating the above procedure while $\alpha$ remains constant $(\alpha=1)$, the following stability regions in $\left(K_{D \theta}, K_{D \varphi}, \beta\right)$ parameters space can be obtained, as shown in Fig. 5. 


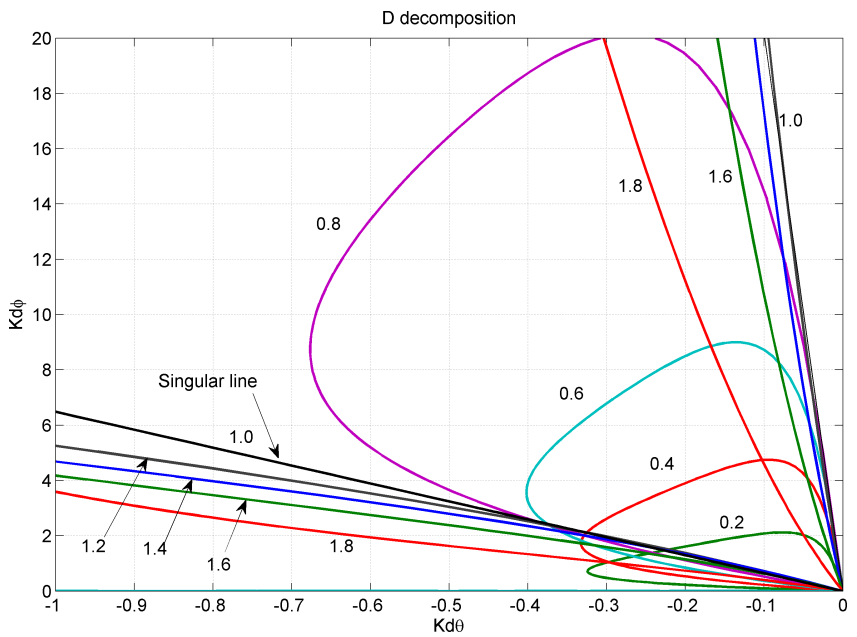

Fig. 4. 2D Stability regions for $\alpha=(0,2), \beta=1$

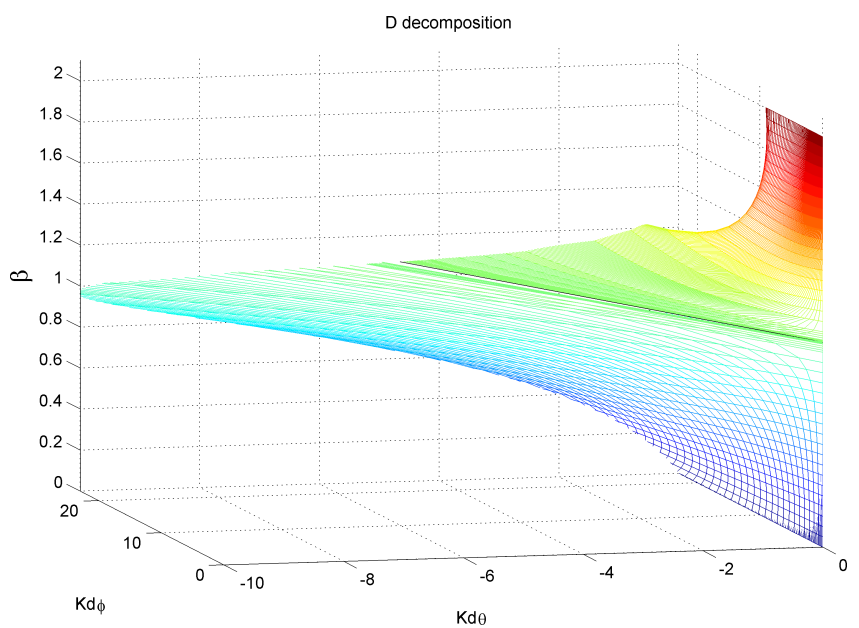

Fig. 5. 3D stability regions for $\beta=(0,2), \alpha=1$

The stability region in a $2 \mathrm{D}$ plot is shown in Fig. 6. It can be seen that the biggest stability region is obtained for $\beta \approx 1$. As in the previous example, singular lines are determined by (37) and for the case $\alpha=\beta=1$. In this example, we could

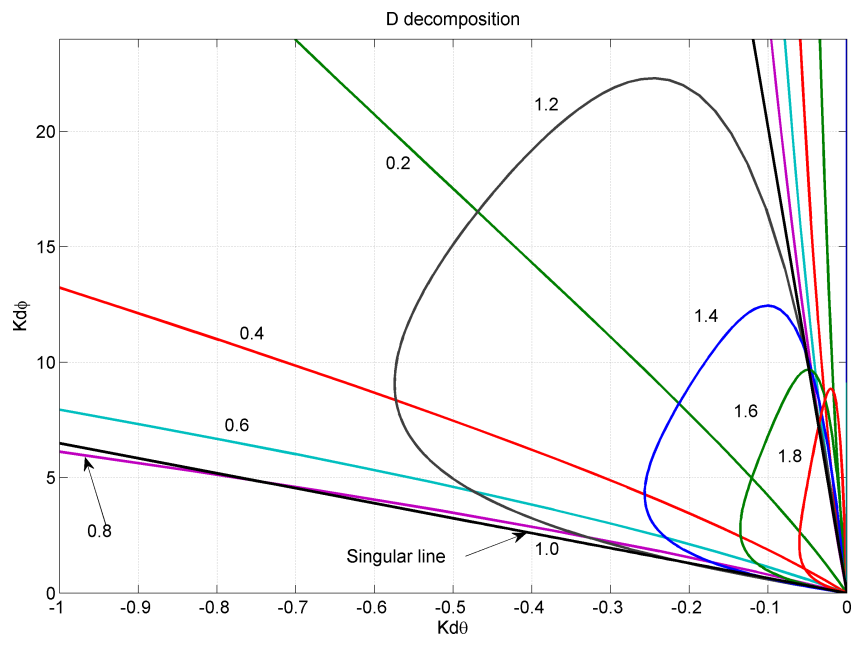

Fig. 6. 2D stability regions for $\beta=(0,2), \alpha=1$ choose any other constant value for $\alpha$ instead of $\alpha=1$. For example, if we choose $\alpha=\alpha^{*}=$ const, we would obtain similar results, except that singular lines would be calculated for $\beta=\alpha^{*}$.

Based on Lyapunov's indirect method theorem, the stability regions obtained in the above examples for the system (16), (17), are the same as for the nonlinear system (11), (12), but only in the small area of the equilibrium point.

As mentioned before, the stability domain $\mathrm{D}(0)$ in all examples is chosen by testing an arbitrary point and checking the stability of polynomial (21). Herein, a simulation is performed to confirm that stability boundaries are well calculated. In Fig. 7 four different points for $\alpha=0.9, \beta=1.1$ are tested using the impulse response.

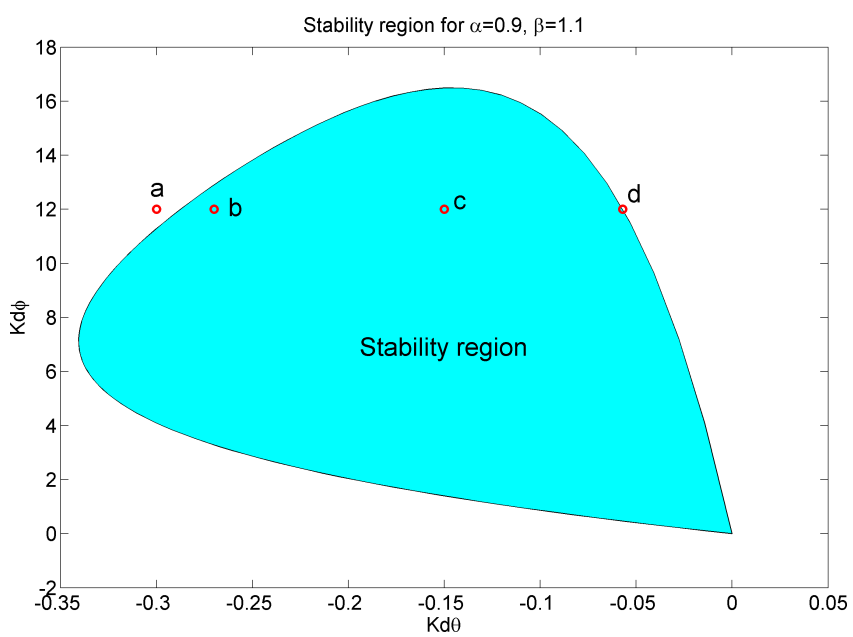

Fig. 7. Stability region testing

Points have been marked as $a, b, c$ and $d$. As we can see, point $a$ lies outside the stability region. If the parameters $\left(K_{D \theta}, K_{D \varphi}\right)$ are determined by point $b$ and $c$, the closed loop system should be stable, while the one with point $d$ should be on the stability margin.

Using the numerical inverse Laplace transformation impulse response of $1 / f(s)$ for each point is obtained. Figure 8 shows the results. In this paper, numerical inverse Laplace transform based on the complex Fourier series approximation is used. Effective numerical algorithms can be developed in for example, Matlab environment. This procedure can be used to invert rational as well irrational or transcendental functions of the complex variable $s$. The required accuracy can be accomplished only at the cost of longer computational time, and without changing the algorithm. Reference [21] is added to explain in more depth the numerical calculation of the inverse Laplace transformation used in this paper.

From Fig. 8 we can see the impulse responses are as expected, which confirms that the stability domain has been properly determined. 


\section{D-decomposition technique for stabilization of Furuta pendulum: fractional approach}
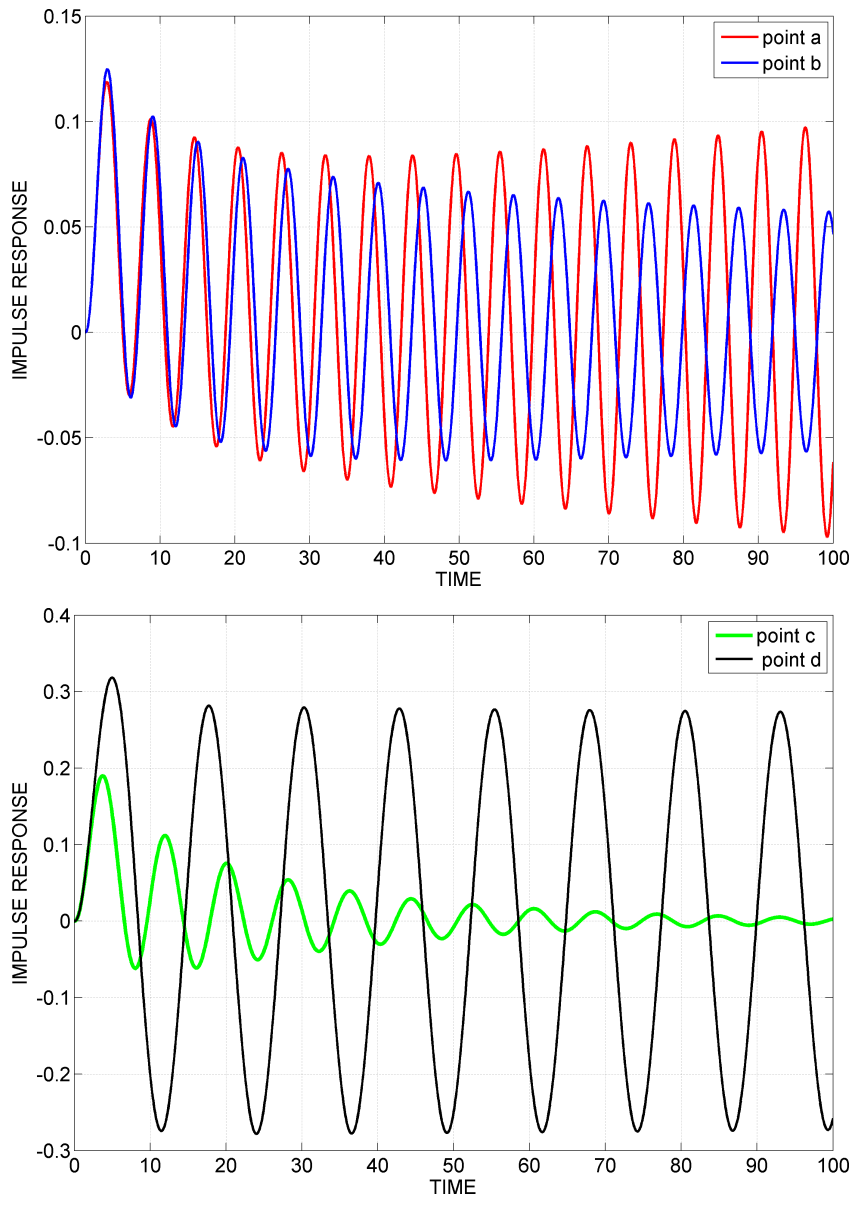

Fig. 8. Impulse response for points $a, b, c$ and $d$

\section{Conslusions}

This paper deals with the stability problem of the inverted pendulum controlled by a fractional order PD controller. Firstly, the mathematical model of Furuta pendulum is derived. Then, a fractional order PD controller is introduced in order to stabilize it. D-decomposition method is used for determining stability region in controller parameters space. Also, the Ddecomposition technique is extended for the linear fractional systems and for the case of linear parameters dependence. Then, the results obtained are used and generalized for the case of nonlinear parameter dependence. In order to confirm that stability boundaries are well calculated certain examples are given and tests are made.

A more detailed analysis of the D-decomposition method for linear fractional order systems will be a subject of future research. More precisely, special attention will be given to the problem of relative stability, since in this paper only absolute stability of the system is considered. Further, generalization of D-decomposition technique for the case of nonlinear parameter dependence will be investigated. Also, transfer from simulation to real laboratory model of Furuta pendulum will be considered in a future work.

Acknowledgements. The authors would like to thank the anonymous reviewers for their helpful and constructive com- ments that contributed to improving the final version of the paper. Authors gratefully acknowledge the support of the Ministry of Education, Science and Technological Development of the Republic of Serbia under the project TR 33047 (P.D.M.) as well as the support by the projects TR 35006 (M.P.L.) and TR 33020 (T.B.Šs).

\section{REFERENCES}

[1] Yu.I. Neimark, "On the problem of the distribution of the roots of polynomials", Dokl. Akad. Nauk SSSR 58, 357-360 (1947), (in Russian).

[2] Yu.I. Neimark, "D-decomposition of the space of the quasipolynomials", Appl. Math. Mech 13, 349-380 (1949), (in Russian).

[3] J.A. Acosta, "Furuta's pendulum: a conservative nonlinear model for theory and practise", Mathematical Problems in Engineering 2010, 742-894 (2010).

[4] I. Podlubny, Fractional Differential Equations, Academic Press, San Diego, 1999.

[5] S.G. Samko, A.A. Kilbas, and O.I. Marichev, Fractional Integrals and Derivatives: Theory and Applications, Gordon and Breach, London, 1993.

[6] K.B. Oldham and J. Spanier, Fractional Calculus: Theory and Applications, Differentiation and Integration to Arbitrary Order, Academic Press, New York, 1974.

[7] I. Podlubny, "Fractional-order systems and $P I^{\lambda} D^{\mu}$ controllers", IEEE Trans. Automatic Control 44, 208-214 (1999).

[8] V. Čović and M.P. Lazarević, Robot Mechanics, Faculty of Mechanical Engineering, Belgrade, 2009, (in Serbian).

[9] M.P. Lazarević, M. Rapaić, and T.B. Šekara, "Introduction to fractional calculus", in: Advanced Topics on Applications of Fractional Calculus on Control Problems, System Stability and Modeling, pp. 3-18, WSEAS Press, Athens, 2014.

[10] M. Cajić and M.P. Lazarević, "Fractional order spring/springpot/actuator element in a multibody system: application of an expansion formula", Mechanics Research Communications 62, 44-56 (2014).

[11] H. Khalil, Nonlinear Systems, Prentice Hall, Upper Saddle River, 2002.

[12] K. Furuta, M. Yamakita, and S. Kobayashi, "Swing-up control of inverted pendulum using pseudo-state feedback", J. Systems and Control Engineering 206 (6), 263-269 (1992).

[13] A. Ruszewski, "Stability regions of closed loop system with time delay inertial plant of fractional order and fractional order PI controller", Bull. Pol. Ac.: Tech. 56 (4), 329-332 (2008).

[14] P.D. Mandić, M.P. Lazarević, and T.B. Šekara, "Fractional order PD control of Furuta pendulum: D-decomposition approach", Proc. IEEE International Conf. on Fractional Differentiation and Its Applications 1, CD-ROM (2014).

[15] P.D. Mandić, M.P Lazarević, and T.B. Šekara, "Ddecomposition method for stabilization of inverted pendulum using fractional order PD controller", Proc. First Int Conf. on Electrical, Electronic and Computing Eng. 1, CD-ROM (2014).

[16] E.N. Gryazina, B.T. Polyak, and A.A. Tremba, "Ddecomposition technique state-of-the-art", Automation and Remote Control 69, 1991-2026 (2008).

[17] T.B. Šekara, Fractional Order Control Systems, Faculty of Electrical Engineering, East Sarajevo, 2011, (in Serbian).

[18] M. Stojić, Continuous Control Systems, Nauka, Belgrade, 1996, (in Serbian). 
P.D. Mandić, M.P. Lazarević, and T.B. Šekara

[19] S. E. Hamamci, "An algorithm for stabilization of fractionalorder time delay systems using fractional-order PID controllers", IEEE Trans. Automatic Control 52, 1964-1969 (2007).

[20] M. Buslowicz "Frequency domain method for stability analysis of linear continuous time fractional systems", in: Recent Advances in Control and Automation, eds.: K. Malinowski and
L. Rutkowski, pp. 83-92, Academic Publishing House Exit, Warsaw, 2008.

[21] L. Brančik, "Numerical inverse Laplace transforms for electrical engineering simulation", in: Matlab for Engineeris - Applications in Control, Electrical Engineering, IT and Robotics, pp. 51-74, InTech, Rijeka, 2011. 